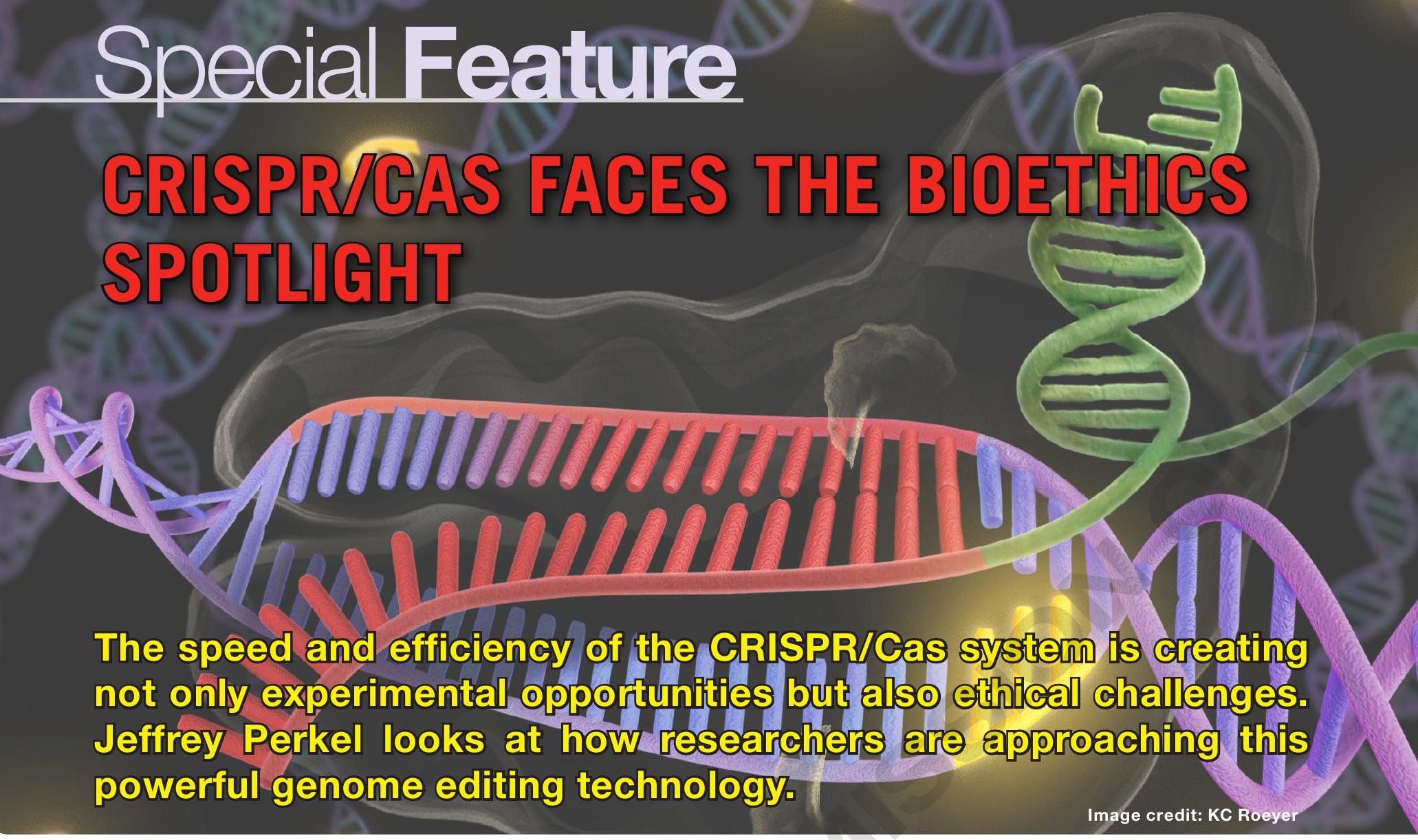

The Three years ago, Jennifer Doudna and Emmanuelle Charpentier launched a molecular biology revolution by unraveling the CRISPR/Cas system. Their seminal paper, published in June 2012 and cited over 900 times according to Google Scholar, describes a mechanism by which the Cas9 enzyme can cleave any segment of DNA, guided by a short RNA molecule complementary to the sequence of interest. Scientists quickly took note and have since exploited the finding to rewrite the genomes of cultured cells and model organisms, tweak gene expression programs, and drive drug development and disease etiology research.

The interesting thing is that, for all the hoopla surrounding CRISPR/Cas9 at the moment, the technique really doesn't do much that researchers couldn't have done before using other technologies. What makes CRISPR/Cas unique is that it is far more accessible and easy to implement. Other nuclease-based genome editing technologies such as zinc finger nucleases and TALENs are more complex systems with protein-based recognition domains. With the CRISPR/Cas9 system, all that is required is the widely available Cas 9 enzyme and an appropriate guide RNA for targeting.

The problem is, such simplicity cuts both ways. On the plus side, essentially anybody with basic molecular biology expertise can use CRISPR for genome editing. On the other hand, anybody can use the technology for almost any purpose.

"We've created CRISPR technologies that are easy enough that people can impulsively do experiments that could have consequences - at a minimum, public relations consequences," suggests Harvard University geneticist George Church, who studies CRISPR.

\section{Ethics classes}

Researchers are working to head off those consequences. In January, 18 researchers, clinicians, ethicists, and lawyers assembled in Napa, California, to discuss what should be done about the real possibility of CRISPR/Cas9 technology being used to modify the genomes of human embryos and germ cells-making heritable genetic changes. The consensus from the meeting, which was published March 19 in a perspective in the journal Science, calls on the scientific community to "strongly discourage" genomic modification of the human germ line (1). Edward Lanphier and Fyodor Urnov, CEO and senior scientist, respectively, at Sangamo BioSciences, and their colleagues independently called for a moratorium on human germline editing in a commentary published online March 12 in the journal Nature (2).

This is not the first time ethical debate over the use of CRISPR technology has emerged; last year researchers 


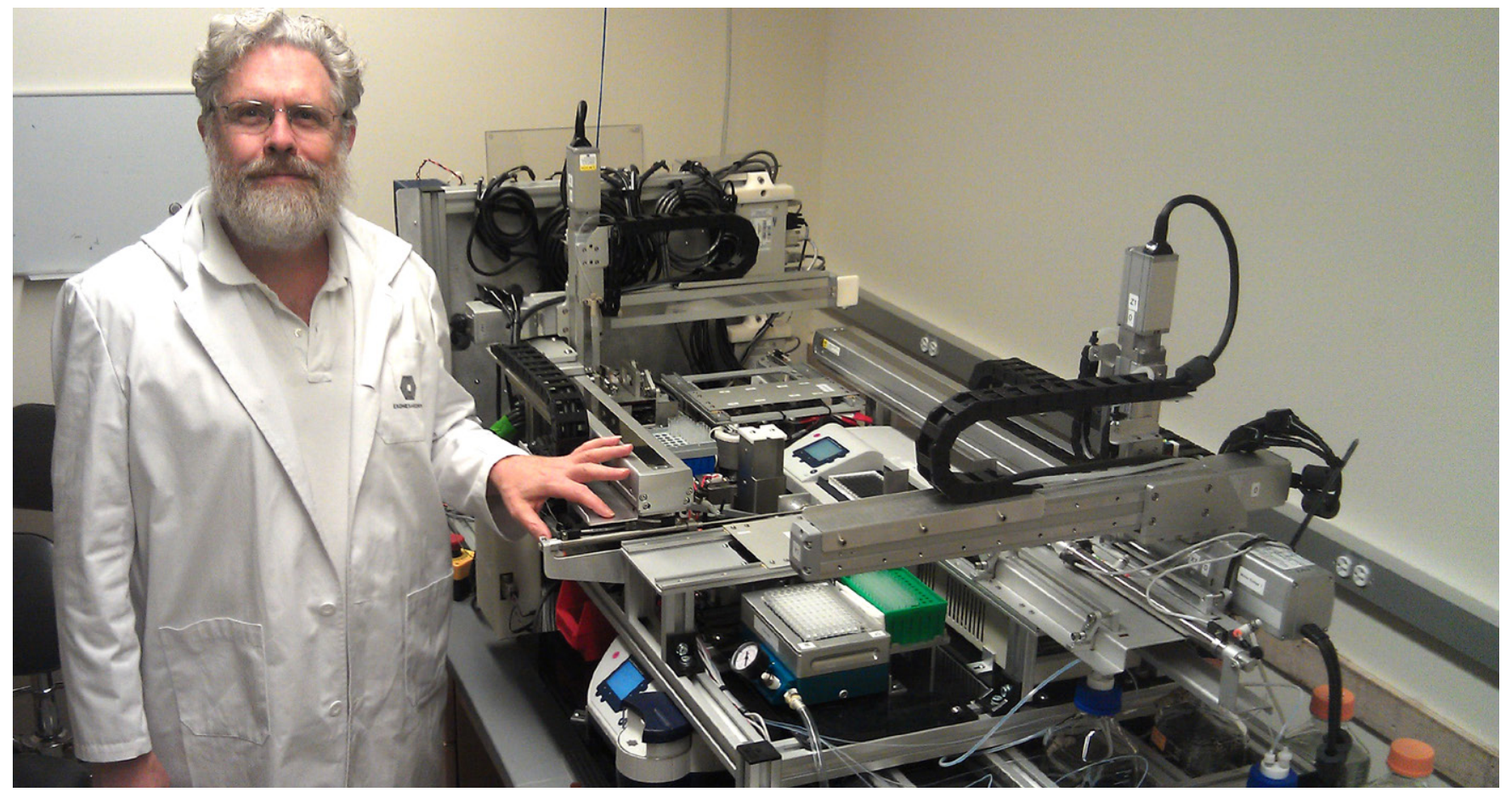

George Church is one of the researchers exploring the applications of CRISPR/Cas9 technology. Credit: Marie Wu.

proposed regulatory and technical solutions to another controversial CRISPR-enabled technology, gene drives, which essentially serve to push a genetic trait through a population in a non-Mendelian fashion $(3,4)$. Among other things, gene drives could be used to control insect pest populations, for instance to reduce the incidence of malaria. One study demonstrating the efficiency of such a system in Drosophila was published alongside the Science perspective in March (5).

Doudna, who organized the Napa conference, says the impetus for the meeting and subsequent perspective article was "the appreciation of the power of the [CRISPR/Cas] technology and the desire to get out in front of that conversation to help people understand it ... and decide on a consensus path forward, if possible."

Lanphier's team's commentary was driven by the recognition that genome-editing technology - whether via CRISPR or some other methodologyis pushing life scientists ever closer to a "line in the biological sand." Indeed, the line already has been crossed. A paper using CRISPR/Cas9 to modify human embryos was published in midApril as this article was going to press. (See Reference 6 and "Breaking News: Germline Editing with CRISPR" following this article.)

\section{History as a guide}

Researchers have manipulated the germ lines of laboratory animals for decades, for instance to make transgenic mice. In 2014, a Chinese team even reported CRISPR modifications in cyanomolgus monkey embryos, "most likely including the germline" (7). But when it comes to humans, genome engineering research has al-

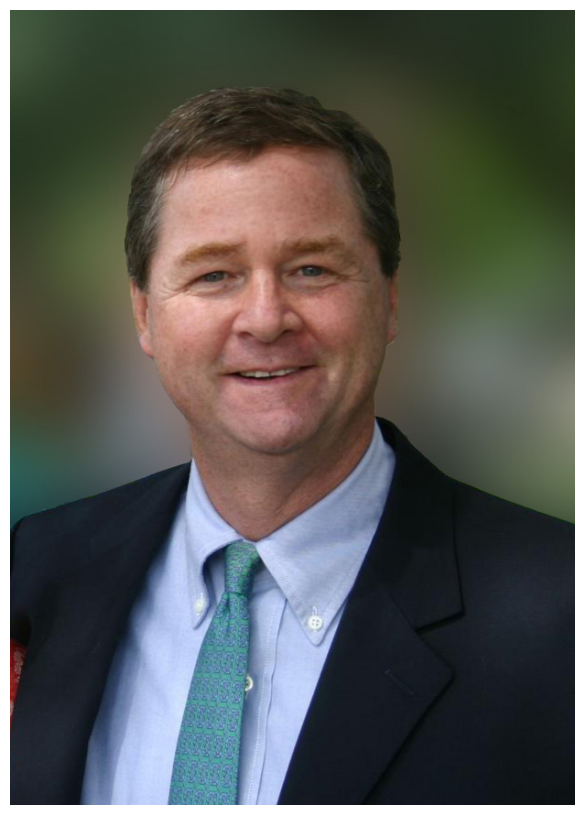

Edward Lanphier, CEO of Sangamo BioSciences, co-authored a commentary in the journal Nature calling for a moratorium on using CRISPR for human germline editing. Credit: Sangamo BioSciences. ways involved modification of somatic (that is, non-heritable) cells. Sangamo is running clinical trials that use zinc finger nucleases to engineer T-cells to be resistant to HIV infection by knocking out the CCR5 receptor, which the virus uses to enter the cells. Although such a treatment may render the recipient resistant to HIV, their children would still be vulnerable to the virus.

Though the Science and Nature articles agreed on the possible dangers of human germline modification, the two reach different conclusions about how to address these risks. Lanphier and his colleagues recommended an outright moratorium while the technical and ethical considerations are hashed out. "Let's pause before we perfect and publish on the Internet a connect-the-dots perfected strategy for making a full human being," Lanphier says. "Let's pause and 


\section{Monitor targeting} efficiency \& specificity in CRISPR/Cas9 experiments

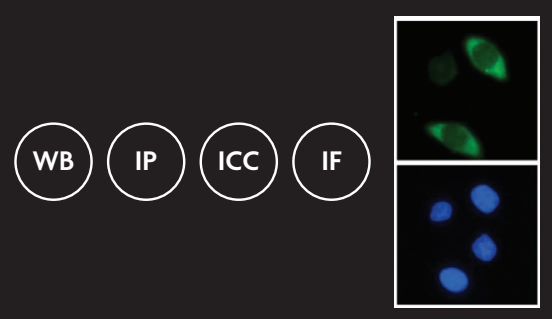

Learn More

www.activemotif.com/Cas9

RCTIVESMOTIF

Enabling Epigenetics Research make sure that there is a rationale for perfecting those techniques in human embryos." At least, he urges, researchers and other stakeholders need to have the discussion-after all, however noble the intentions of those who first use the technology, others may use it to drive any application they desire. "Genome-editing platforms are agnostic to the gene that you can target and modify," he notes.

Doudna and her colleagues from Napa, on the other hand, see value in the basic research possibilities, for instance for the study of mechanisms of DNA repair, and for that reason stop short of calling for an outright moratorium. Instead, she says, progress should be carefully considered and approved on a case-by-case basis. "Only by doing research will we have the data to make decisions about any potential future applications of the technology."

Church argues that a de facto moratorium already is in place, as researchers proposing human clinical trials must demonstrate safety and efficacy in culture and animal models first to the satisfaction of bioethics review boards and regulatory agencies.

with questions surrounding in vitro fertilization. Then, as now, researchers and ethicists argued over potential pitfalls, and asked whether the payoff for infertile couples was worth the risk. Once Louise Brown, the first "test-tube baby," was born, the discussion, he says, was flipped on its head. "Now, rather than it being unethical to do any experiments along these lines, it became unethical to deprive couples of clinical access to these advanced reproductive technologies."

Whether genome editing ultimately achieves a similar milestone is anybody's guess. But Doudna appreciates the comparison. At Napa, she says, one of the attendees observed that "maybe there will be a time when it would be considered unethical not to do [genome editing in the germ line] for certain applications. And everyone said, 'huh, that's a different way of thinking about it."'

In the short-term, the life science community must come together and decide how best to move forward. A more inclusive follow-up to the Napa meeting is already in the works. "It's under very active discussion from many parties," Doudna notes.

\section{Where are we heading?}

It isn't entirely clear that germline editing with CRISPR/Cas offers compelling advantages for human health beyond existing technologies, such as pregenetic diagnosis during in vitro fertilization. And there remain substantial technical hurdles to overcome even if it does, such as the possibility for offtarget or unintended effects. Church (who was a co-author the Doudna article, though he did not attend the Napa meeting) posits the example of a person with two balancing mutations. Researchers could conceivably fix one problem with the genome editing technology, only to create another for the patient. In the end, meticulous research would be required to identify and resolve all of these different possibilities.

Church likens the current debate to the 1970s, when scientists grappled

\section{References}

1. Baltimore, D., et al. 2015. A prudent path forward for genomic engineering and germline gene modification. Science, 348:36-38.

2. Lanphier E., et al. 2015. Don't edit the human germ line. Nature, 519:410-411.

3. Esvelt, K.M., et al. 2014. Concerning RNA-guided gene drives for the alteration of wild populations. eLife, 17:e03401. Science. 345:626-628.

5. Gantz, V.M. and Bier, E. 2015. The mutagenic chain reaction: A method for converting heterozygous to homozygous mutations. Science. 348:442-444

6. Regalado, A. "Engineering the perfect baby," MIT Technology Review. March 5, 2015.

7. Niu, Y., et al. 2014. Generation of genemodified cynomolgus monkey via Cas9/ RNA-mediated gene targeting in one-cell embryos. Cell. 156:836-843.

Written by Jeffrey Perkel, Ph.D. 罝

BioTechniques 58:223-226 (May 2015) doi: 10.2144/000114284
4. Oye, K.A., et al. 2014. Regulating gene drives. 


\section{Breaking News: Germline Editing with CRISPR}

As this article was set to go to press, a team of Chinese researchers published the first report of human germline editing (1).

Led by Junjiu Huang at Sun Yat-sen University in Guangzhou, China, the team used CRISPR/Cas9 to edit the human beta-globin (HBB) gene in tripronuclear embryos (fertilized by two sperm) produced by in vitro fertilization. These embryos, the authors say, could never be viable.

The team injected 86 tripronuclear embryos with mRNAs encoding Cas9 and GFP, a guide RNA targeting HBB, and a single-stranded oligonucleotide encoding six "silent" mutations as a repair template. Forty-eight hours later, 71 embryos remained viable, of which 59 were GFP-positive. Analysis of 54 of those embryos revealed the HBB gene had been targeted successfully in 28 of them, but only 4 were modified through homologydependent repair using the supplied template, and those yielded genetic mosaics. Seven had used an endogenous gene related to HBB as the template, while 17 were repaired using non-homologous end-joining. Exome sequencing analysis of six successfully targeted embryos identified off-target mutations in the transthyretin and C1QC coding sequences.

"[O]ur data underscore the need to more comprehensively understand the mechanisms of CRISPR/Cas9-mediated genome editing in human cells, and support the notion that clinical applications of the CRISPR/Cas9 system may be premature at this stage," the authors conclude.

In a statement emailed to BioTechniques, Jennifer Doudna, writes, "Although it has attracted a lot of attention, the study simply underscores the point that the technology is not ready for clinical application in the human germline. And that type of use of the technology needs to be on hold pending a broader societal discussion of the scientific and ethical issues surrounding such use."

From a technical standpoint, George Church says in an email that his group has published methods to minimize off-target effects, which Huang's group cited in their article but did not employ. The authors, he says, argue that off-target effects seem to be less-prevalent in embryonic cells than cancer cells, and for that reason, this kind of experiment could help more accurately assess safety and efficacy without endangering clinically viable embryos. "While it would be nice if they had tried the best technology, the results are still quite relevant to the discussion."

But he also notes that this study focused on an application for which germline editing may be ideally suited. "[T]he HBB gene is representative of many diseases in which both parents are homozygous for deleterious alleles ... and hence other (non-genome editing) methods would be considered inapplicable (like IVF-PGD [in vitro fertilization-preimplantation genetic diagnosis]) or possibly less safe and/or less effective (somatic gene therapy with mosaic off-target)." --J.P.

1. Liang, P., et al. 2015. CRISPR/Cas9-mediated gene editing in human tripronuclear zygotes," Protein Cell. doi:10.1007/s13238-015-0153-5 Apr 18. [Epub ahead of print].

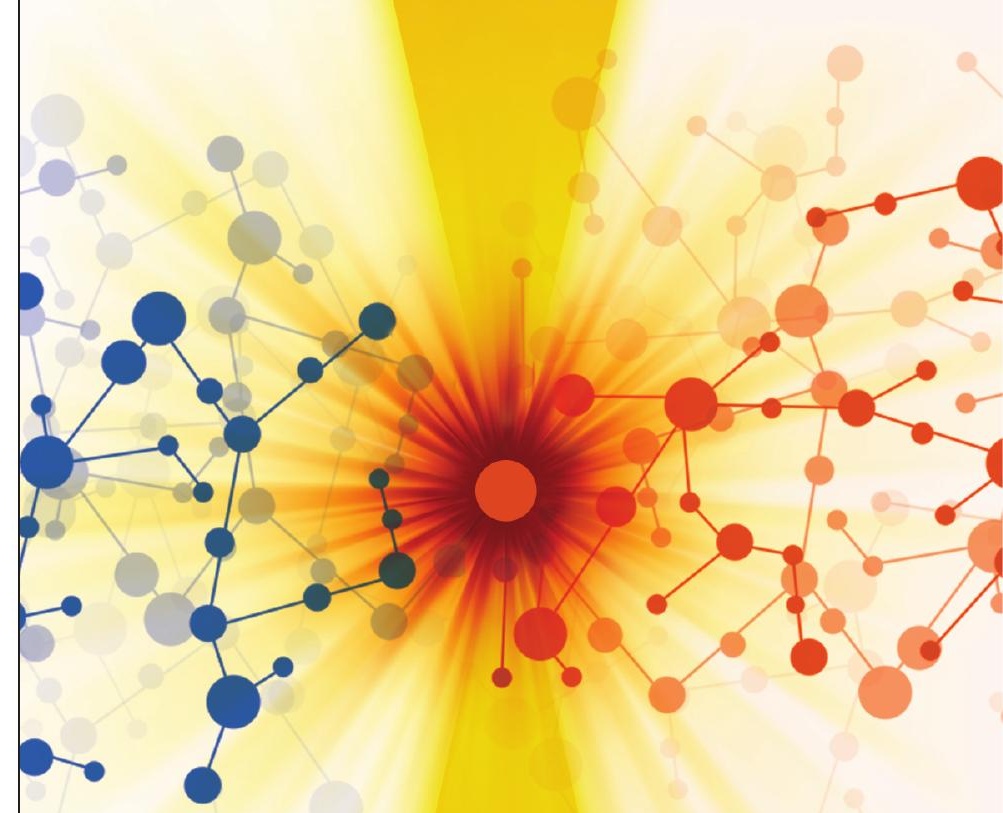

\section{SINGLE MOLECULE COUNTING IMMUNOASSAY TECHNOLOGY: Bringing novel biology to light}

\section{Putting the power of SMC ${ }^{\mathrm{TM}}$ technology to work in every lab.}

Singulex's proprietary digital Single Molecule Counting immunoassay technology allows scientists to measure proteins with unparalleled precision, enabling quantification at low and high levels of expression with dynamic range $>4$ logs.

The flexible Erenna ${ }^{\circledR}$ Immunoassay System acquires data from both plate-based and bead-based assays, providing a choice of format depending on your quantification requirements, at a price affordable for any research program.

\section{Life Science Products and Custom Services}

Erenna Immunoassay System

Plate \& Bead Immunoassay Kits

Custom Assay Development Services

Contract Sample Testing Services

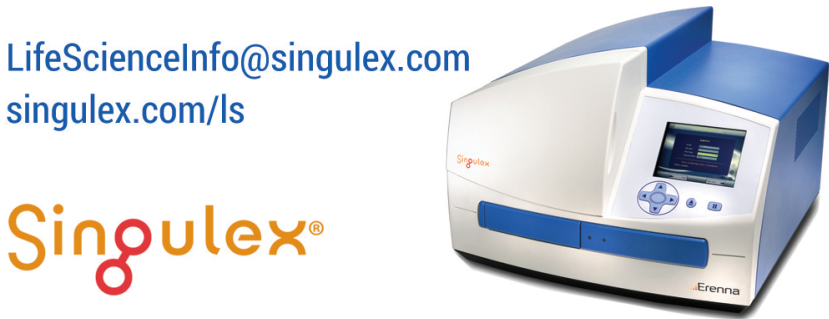

(c) Copyright 2015 Singulex, Inc. All Rights Reserved. Singulex, SMC and Erenna are trademarks or registered trademarks of Singulex, Inc. For Research Use Only. Not for use in diagnostic procedures. 